\section{Hot dogs - What triggers fan the flames of heat related illness for UK dogs?}

\author{
Emily Hall ${ }^{1}$, Anne Carter ${ }^{1}$, Dan O'Neill ${ }^{2}$ \\ 1 Nottingham Trent University, Nottingham, United Kingdom \\ 2 Royal Veterinary College, London, United Kingdom
}

\section{OBJECTIVES}

Current heat related illness awareness campaigns focus on hot cars as the dominant trigger, but other causes could be more important. This study aimed to explore common triggers for heat related illness in dogs under primary veterinary care in the UK.

\section{METHODS}

Dogs with heat related illness events during 2016 were identified from VetCompass ${ }^{T M}$ first-opinion clinical records. Inciting events and mortality data were extracted to compare common triggers. Logistic regression analysis explored fatality risk.

\section{RESULTS}

From 905,543 dogs 1222 cases were identified, some with multiple events. Overall, $30.2 \%$ of events had no specific cause reported. Of the remaining 879 events, the most common triggers were exercise $74.2 \%$, exposure to a hot outdoor environment $13.0 \%$, confinement in a hot car $5.2 \%$, treatment by a veterinarian or groomer $4.6 \%$ and confinement in a hot building $3.1 \%$. The 2016 fatality rate of heat related illness cases was $14.2 \%$. Of the known causes, only cases following entrapment in a hot building had increased odds of death compared to cases following exercise (odds ratio $6.6,95 \% \mathrm{Cl} 2.6-16.6$ ).

\section{STATEMENT (CONCLUSIONS)}

This study highlights exercise as the most common cause of heat related illness in UK dogs, accounting for fourteen times as many cases as hot cars; entrapment in a hot building had six times the odds of death. These findings suggest that we need to rethink heat related illness education strategies, veterinarians should alert owners to the hazards of exercising dogs in hot weather or leaving them in hot buildings in addition to annual "dogs die in hot cars" campaigns.

\section{Unusual presentation of ulcerative necrotizing stomatitis and rhinitis with neutrophilic- eosinophilic- granulomatous inflammation and possible vasculitis in a dog}

\section{Perrine Henry, Andrew Perry, David Mackenzie}

Eastcott Referrals, Swindon, United Kingdom

\section{OBJECTIVES}

To present an unusual case of probable immunemediated necrotizing stomatitis and rhinitis.

\section{METHODS \\ Case report.}

\section{RESULTS}

A 2-year-old female springer spaniel was referred for severe recurrent stomatitis and unilateral nasal discharge. Gingival lesions were treated for 17 months prior to presentation with extended courses of antibiotics, NSAIDs, local antiseptic, surgical debridement and teeth extraction which resulted in only temporary improvement.

Oral examination revealed extensive multifocal necrotizing ulcers with pseudomembrane on the buccal mucosa with no evidence for periodontal disease. Diagnostic investigation including blood test (CBC, biochemistry, infectious disease screening), CT scan, and biopsies of oral, para-oral and nasal tissue (for histopathology, bacteriology and fungal culture) gave a diagnosis of neutrophilic, eosinophilic, granulomatous stomatitis and rhinitis. The partial response to the above treatment, and the histopathology findings, suggested an immune-mediated process. Treatment with prednisolone $(0.75 \mathrm{mg} / \mathrm{kg}$ BID) resulted in a clinical improvement within 2 days. However, tapering of steroids (to $0.3 \mathrm{mg} / \mathrm{kg}$ BID) caused relapse of clinical signs. Subsequent biopsies suggested an underlying neutrophilic vasculitis. Accordingly, treatment with Pentoxifylline $(18.75 \mathrm{mg} / \mathrm{kg}$ BID) and ciclosporin $(5 \mathrm{mg} / \mathrm{kg} /$ day) was added, which resulted in complete remission for 6 months. Subsequent relapse 